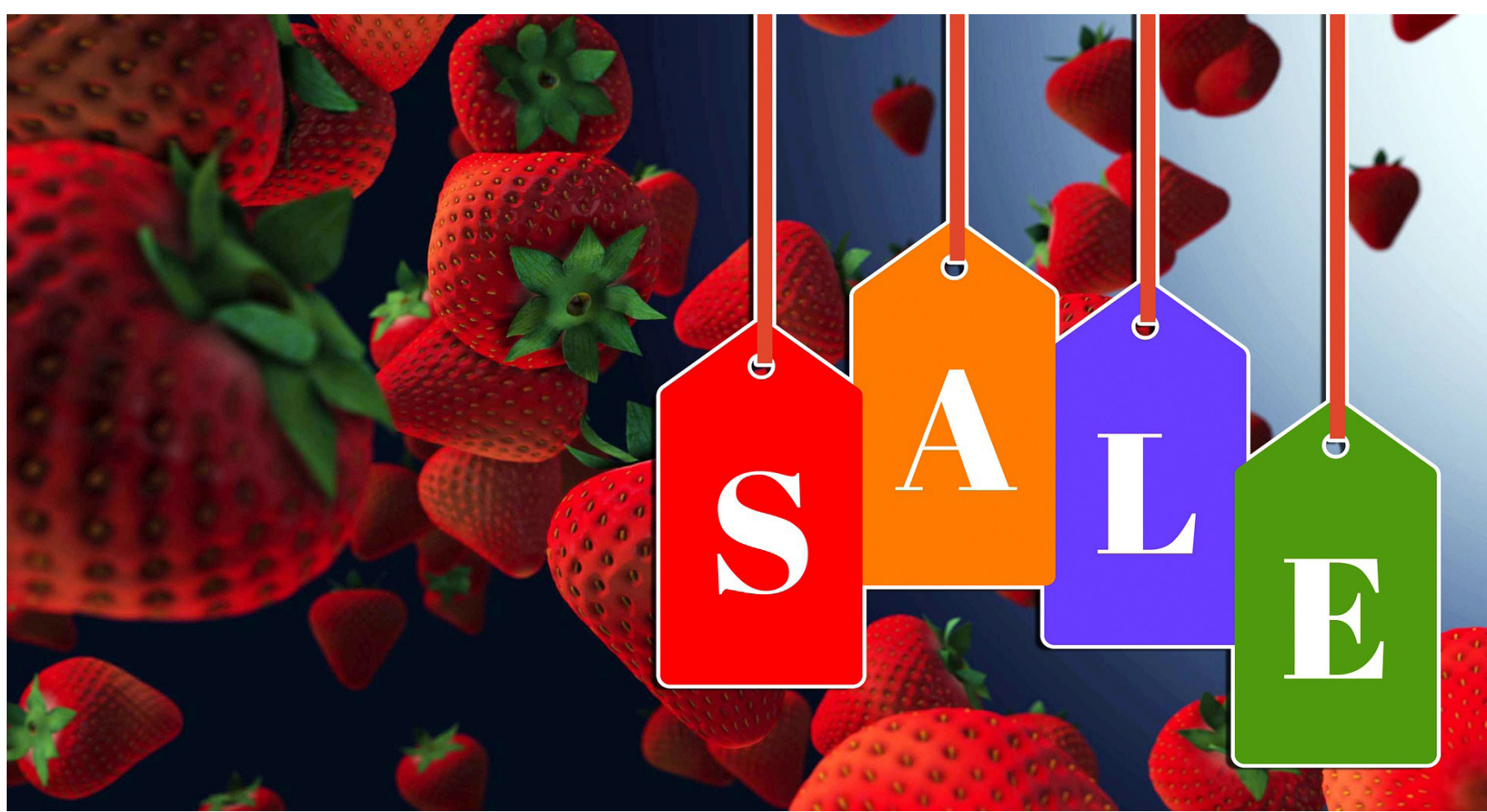

\title{
Laproducciónagrícoladeloscampesinos deToledo
}

\section{The agricultural production of Toledo's}

\section{Yuleisi Pilar Borja-Villalba}

Estudiantes de Negocios Internacionales yp.borja@fesc.edu.co Fundación de Estudios Superiores Comfanorte, Cúcuta, Colombia

Betsabec Urbina-Pulido

Estudiantes de Negocios Internacionales, b.urbina@fesc.edu.co Fundación de Estudios Superiores Comfanorte, Cúcuta, Colombia
Sharyn Nataly Hernandez- Fuentes

Maestría en Dirección y Gestión de Centros Educativos sn_hernandez@fesc.edu.co, Fundación de Estudios Superiores Comfanorte, Cúcuta, Colombia 


\section{Resumen}

La población rural en Colombia está conformada por campesinos, en su gran mayoría considerados pobres, debido a la falta de recursos percibidos y a los ingresos generados por las ayudas del gobierno nacional. La población rural de Toledo Norte de Santander, en el 2015 se vio seriamente afectada por los niveles altos de pobreza a causa del desplazamiento y la falta de oportunidades para poder trabajar y cultivar sus tierras. El propósito de la presente investigación fue Identificar las falencias que tienen los campesinos de Toledo N. de S. en su proceso de producción en donde se utilizó como metodología enfoque cuantitativo explicativo por que muestra una descripción como información crucial a una solución posible a la problemática existente en la formación académica de los campesinos arrojados por las encuestas realizadas a los agricultores de Toledo Norte de Santander. Tuvo como resultados información necesaria y suficiente que permite llegar a conocer más fondo la problemática que hay en la falta de educación hacia los campesinos de Toledo Norte de Santander teniendo en cuenta las falencias que hay en la falta de compromiso de parte del gobierno nacional que no tiene presente las necesidades de los ciudadanos y campesinos.

Palabras clave: campesinos, ayudas gubernamentales, café, formación, Toledo.

\section{Abstract}

Problem: The rural population in Colombia is made up of peasants, the vast majority of whom are considered poor, due to the lack of resources and the income generated by national government aid. The rural population of Toledo Norte de Santander in 2015 was seriously affected by high levels of poverty due to displacement and lack of opportunities to work and cultivate their land. Objective: To identify the shortcomings of the peasants of Toledo N. de S. in their production process. Methodology: The following research is explanatory because it shows a possible solution to the existing problem in the academic training of the peasants thrown out by the surveys carried out on the farmers of Toledo Norte de Santander. Results: Once this research has been carried out, we have enough and necessary information that allows us to get to know more deeply the problem of lack of education towards the peasants of Toledo Norte de Santander, taking into account the shortcomings in the lack of commitment On the part of the national government that does not have in mind the needs of citizens and peasants.

Keywords: Peasants, government aid, coffee, training, Toledo. 


\section{Introducción}

La educación en Colombia específicamente en las zonas rurales es un asunto de equidad y desarrollo para el país, las regiones y los ciudadanos, incluidos quienes viven en las grandes ciudades. El departamento de Norte de Santander tiene una superficie de $21.987 \mathrm{Km} 2$, equivalente al $1.93 \%$ del área total de Colombia. Toledo a su vez posee una superficie de 157.790,95 Ha que equivalen al $7.176 \%$ de la superficie total del departamento. Al nombrar a Toledo como una zona rural altamente golpeada por la violencia y la falta de oportunidades sumándole a estas situaciones el constante cambio climático, se observa que se realizó un estudio donde se habló de cómo se podría hacer más eficiente y sostenible la economía del municipio. Así mismo se conoce que Toledo tiene mayor producción agrícola en café, caña panelera, cítricos, plátano, morón, frijol y maíz. Gracias a esto podrá llegar a prepararse como uno de los municipios más exportadores del país. Sin embargo, aunque tiene mucho capital por explotar una de sus principales realidades son las dificultades con las que trabajan pese a que no cuentan con maquinaria, ni nuevas tecnologías que le faciliten el desempeño de sus labores agrícolas.

\section{Materiales y métodos}

Al tratarse de una investigación explicativa que muestra una posible solución a la problemática existente en la formación académica de los campesinos arrojados por las encuestas realizadas a los agricultores de Toledo Norte de Santander.

Se efectuó la encuesta a los habitantes del municipio de Toledo, los cuales se les aplicó la muestra a 100 personas de diferentes edades ubicadas en los sectores agrícolas del municipio.

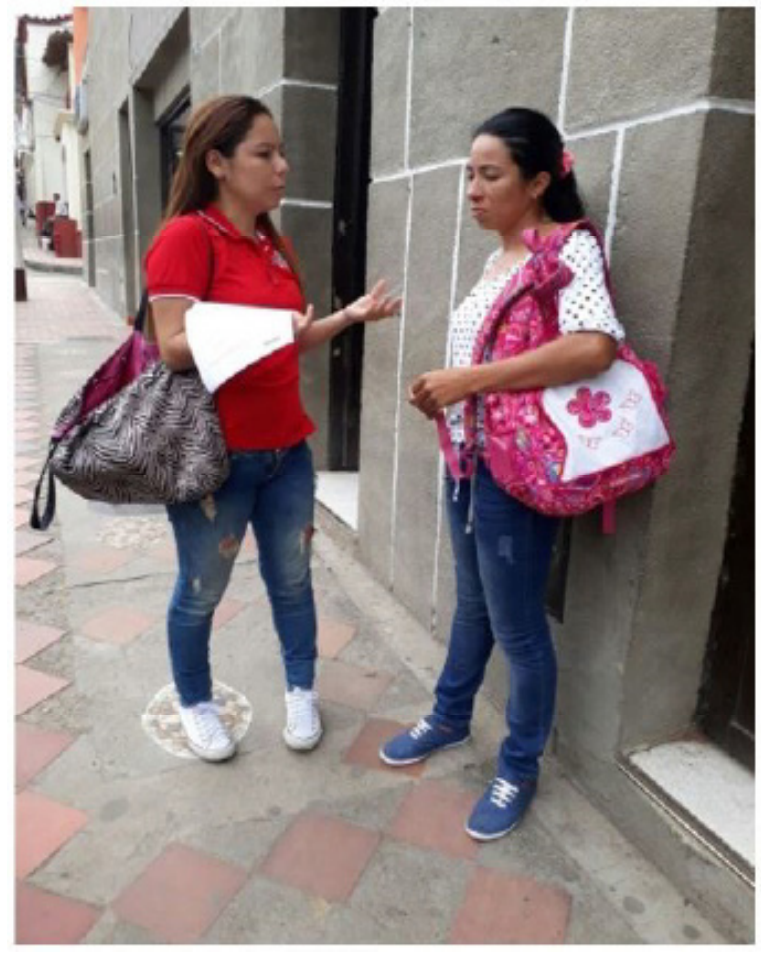

Figura 1. Trabajo de campo

Fuente (propia del investigador).

\section{Resultados y discusión}

Para poder identificar las falencias de los agricultores de Toledo N. de S. en el proceso de producción, se habló con los dueños de predios y gente del común y se llegó a conocer que sus vías tienen un grave deterioro y gran abandono por parte del estado, debido a esto se les dificulta la entrada y salida de su producción y posibles ayudas que puedan ofrecerles el gobierno. Posteriormente necesitan más información y apoyo a sus cultivos. 


\section{8}

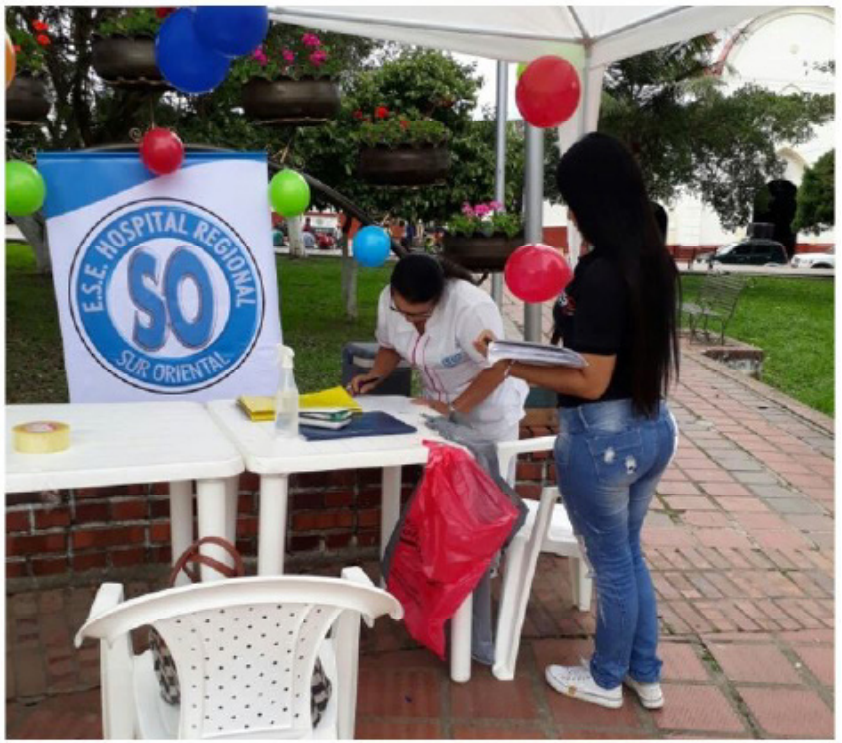

Figura 2. Trabajo de campo

Fuente (propia del investigador)

A pesar que los cafeteros reciben capacitaciones para su producción no es suficiente, puesto y hay más cultivos como la caña, la cebolla, la habichuela etc, que necesitan ser supervisados y llevar el mismo proceso de capacitación para poder tener buenos resultados y así posicionar sus productos nacionales e internacionalmente que son sus metas a futuro. Ante la situación planteada debemos Establecer convenios con el gobierno departamental y nacional con el fin de Financiar las obras en vías, y de mitigación de los riesgos a los cuales está expuesto el Municipio de Toledo, la participación comunitaria será fundamental en las estrategias y las acciones específicas de reducción de riesgo en todos los sectores del municipio de Toledo.

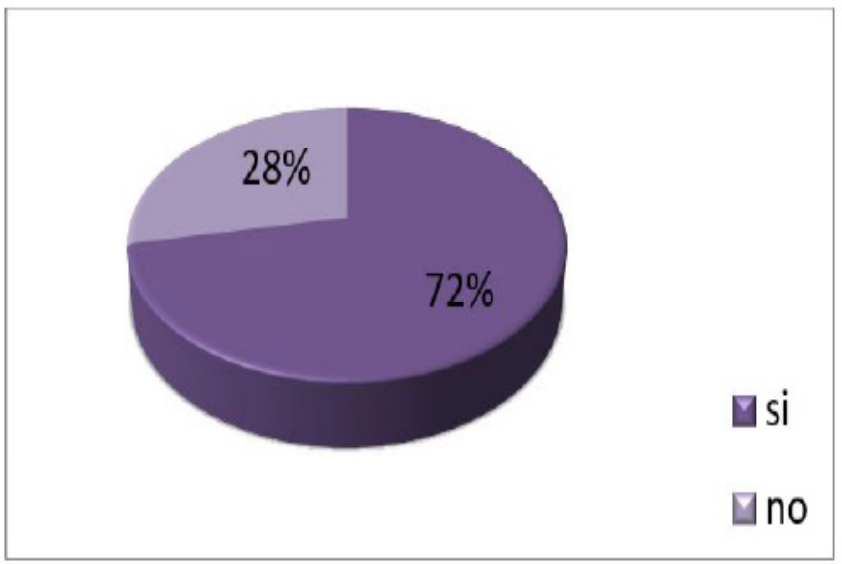

Gráfica 1. Problema de educación

Fuente: Elaboración propia

Como se evidencia en la Grafica 1 la población encuestada respondió que el municipio de Toledo si tiene una problemática de educación con un $72 \%$ por no contar con la orientación y preparación adecuada para el manejo de sus cultivos.

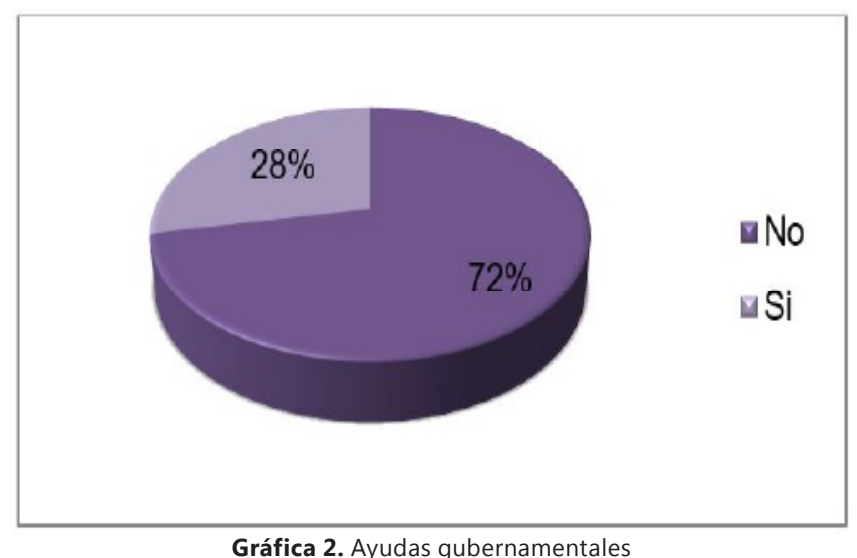

Fuente: Elaboración propia

En la Grafica 2 se puede observar que la población encuestada respondió que No conocen ayudas del gobierno nacional dadas al agricultor, sin embargo un $29 \%$ de los encuestados dijo que si las conocían y que eran capacitaciones dadas a los cafeteros, de igual forma mostraron inconformidad con estas ayudas.

Conclusiones 
Es evidente que la población de Toledo tiene muchas falencias en cuanto a tierras se refiere vías de transporte y falta de educación para el campesino, se escuchó a la población y a dueños de predios, que sería factible para los ciudadanos que tienen esta problemática, establecer convenios con el gobierno departamental y nacional con el fin de Financiar las obras de mitigación de los riesgos a los cuales está expuesto el Municipio de Toledo.

Se observaron falencias por falta de compromiso de parte del gobierno nacional que no tiene presente las necesidades de los ciudadanos y campesinos.

\section{Referencias}

Villarreal, N. (s.f.). Desarrollo rural género y ciudadanía. Recuperado de http://www.bdigital. unal.edu.co/40128/1/Desarrollo\%20rural\%20 genero $\% 20 \mathrm{y} \% 20$ ciudadania.pdf

Javier Serrano Ruiz, Luis Ramiro López, Manual para la formulación y ejecución de planes de educación rural. Recuperado de http://www. mineducacion.gov.co/1759/articles-329722_ archivo_pdf_Manual.pdf

Plan de desarrollo territorial del municipio de Toledo, tomado de http://www.sisubregionalns. gov.co/files/sid_Desarrollo_territorial/PMD/ SurOriental/PDM_TOLEDO_2016-2019.pdf

Departamento Nacional de Estadística. (2019). Disponible en http://www.dane.gov.co/

Tomado de la pagina oficial http://toledonortedesantander.gov.co/index.shtml 